\title{
Local Anaesthetics: What Can We Expect More than Pain Relief?
}

\author{
Iveta Golubovska*, Peteris Studers** and Indulis Vanags*** \\ *Hospital of Traumatology and Orthopaedics, Riga, Latvia \\ **Riga Stradins Univesity, Research Laboratory of Traumatology and Orthopaedics, Latvia \\ ***Riga Stradins University, Department of Anaesthesiology and Reanimatology, Latvia
}

\begin{abstract}
Summary
This problem - solving article considers positive and negative non-analgesic actions of local anaesthetics, which may play a significant role in patient morbidity and mortality. Direct impact on inflammatory and immune substances and cells leads to antibacterial, antiinflammatory, immunomodulating and systemic functions of numerous local anaesthetics. Local anaesthetics used perioperatively locally in continuous infusions or in systemic circulation reach and maintain safe and effective rates, but myotoxic and tissue growth inhibiting effect should be taken in account.
\end{abstract}

Key words: anti-inflammatory, immune response, local anaesthetics, systemic analgesia.

Abbreviations: CNS - central nervous system, BP - bupivacaine, hPMNs - human polymorphonuclear neutrophils, LA - Iocal anaesthetics, LD - lidocaine, LP - lysophosphatide, LPA - lysophosphatidic acid, MP - mepivacaine, NK - natural killer cells, NSAID non-steroidal anti-inflammatory drug, PG E2 - prostaglandin E2, RP - ropivacaine.

\section{INTRODUCTION}

Local anaesthetics (LA) are used widely in anaesthesiology and pain therapy-not only for acute pain, such as during surgery, but also for the management of postoperative and chronic pain. In patients with different (epidural, spinal, wound, plexus, nerve) catheter placement, regional anaesthetics provide a sufficient analgesic effect.

LA are used to facilitate analgesia through their inhibition of sensory nerve conduction. As sodium channel blockers, LA inhibit the propagation of action potentials along nerve axons participating in the transmission of pain signals through the peripheral and central nervous systems. Aside from sodium channel inhibition, it is now becoming clear that LA have actions on other important cellular components.

LA have antibacterial actions and beneficial effects on inflammatory responsessuchasinflammatorylunginjury, increased microvascular permeability, and myocardial ischemia/reperfusion. Smaller concentrations of LA that is currently used may maintain anti-inflammatory effect with decreased risk of toxicity.

There is growing body of evidence that LA have immunological properties in addition to their direct anaesthetic activity, what could be very important in host defence reactions, and regional anaesthesia probably may stop metastatic dissemination after tumour surgery.

\section{Antibacterial properties}

Since the introduction of cocaine in 1884 , LA have been used as a mainstay of pain relief. However, numerous studies over the past several decades have elucidated the supplemental role of LA as antimicrobial agents. In addition to their anaesthetic properties, medications such as bupivacaine (BP) and lidocaine (LD) have been shown to exhibit bacteriostatic, bactericidal, fungistatic, and fungicidal properties against a wide spectrum of microorganisms. We may found in the literature several studies with different LA used in different concentrations used for established pathogens.

Antimicrobial activity of BP against 10 microbial strains was studied. The strains tested were Escherichia Coli, Pseudomonas Aeruginosa, Staphylococcus Aureus, Staphylococcus Epidermidis, Streptococcus Pneumoniae etc. It was found that BP at concentration of 0.25 $\%$ inhibited growth of the sensitive S. Epidermidis, S. Pyogenes, and S. pneumoniae and all the others at the concentration of $0.5 \%(26)$.

It was investigated the antimicrobial effects of different concentrations of RP, BP, LD and prilocaine on Escherichia Coli, Staphylococcus Aureus, Pseudomonas Aeruginosa and Candida Albicans. Ropivacaine (RP) did not inhibit any of the microorganisms tested. BP reduced the viable cells of P. Aeruginosa at $0.5 \%$ and $0.25 \%$ solutions. LD $5 \%$ and $2 \%$ and prilocaine $2 \%$ dilutions reduced the viable cells of all microorganisms tested (2).

It was hypothesized that LA have time-dependent effects on phagocytosis of S. Aureus, oxidative burst, and CDllb expression by human neutrophils (25). Host defence mechanisms are affected and the ability to eliminate bacteria might be reduced. LD and BP inhibited neutrophil functions in a time-dependent manner. Results indicate that in a whole blood model, time-dependent effects of local anaesthetics affect key neutrophil functions necessary for bacterial elimination. But these effects only occur at concentrations that are unlikely to be routinely attained in the clinical setting. Review under antimicrobial properties of a LA (19) was conducted using MEDLINE. Studies testing the effect on microbial growth inhibition of LA alone and 
in combination with other agents, such as preservatives and other medications, as well as the effect of conditions such as concentration and temperature, were included for review. Outcome measures included colony counts, area under the curve and time kill curve calculations, minimum inhibitory concentrations, and post-antibiotic effect. Multiple LA at the concentrations typically used in the clinical setting (e.g., BP $0.125 \%-0.75 \%$; LD $1 \%-3 \%)$ inhibit the growth of numerous bacteria and fungi under various conditions. Different LA showed various degrees of antimicrobial capacity; BP and LD, for example, inhibit growth to a significantly greater extent than does RP. Greater concentrations, longer exposure, and higher temperature each correlate with a proportional increase in microbial growth inhibition. Therefore LA may be weighted as adjunctive to traditional antimicrobial therapy. And also we should take in account a positive message of a relative safety of epidural, plexus and wound catheters.

\section{Antiinflammatory properties}

LA modulate inflammatory responses and may therefore be potentially useful in attenuating perioperative inflammatory injury (13). Clinically relevant concentrations of LA inhibit several actions of the phospholipid mediator lysophosphatidic acid (LPA) on human polymorphonuclear neutrophils (hPMNs). The selective inhibition of LPA-induced priming (15) is probably important, because the priming process is a critical component of hPMN mediated tissue injury both in vitro and in vivo. RP is a relatively new, long acting aminoamide local anaesthetic that is structurally similar to BP and has relatively low CNS and cardiotoxicity compared to BP. RP inhibits platelet-activating factorinduced priming of hPMNs (14). Local wound infiltration of RP results in a longer duration of action compared to BP, because of direct vasoconstrictive effect (29). The vasoconstrictive properties of RP are not unique, other amide local anaesthetic as LD, has similar properties. It is described that vasoactivity of RP could be reduced by using inhibitors of lipoxygenase and phospholipase $\mathrm{A}^{2}$ (6).

Irrigation of a wounds or synovia with small concentrations of a LA maintains good anti-inflammatory effect due to preventing of neurogenic plasma extravasation (24). BP in clinically relevant concentrations inhibits of prostaglandin (PG) E2 receptor functioning in cultured cells (17). BP affects the membrane receptor itself and also the intracellular pathway. The effect is most likely due to modulation of $\mathrm{G}$ protein or phospholipase $\mathrm{C}$ function.

Accumulating data suggest however that LA posses a wide range of anti-inflammatory actions through their effects on cells of the immune system, as well as on other cells, e.g. microorganisms, thrombocytes and erythrocytes. The potent anti-inflammatory properties of LA, superior in several aspects to traditional antiinflammatory agents of the NSAID and steroid groups and with fewer side-effects, would encourage clinicians to introduce them in the treatment of various inflammation-related conditions and diseases. They have proved successful in the treatment of burn injuries, interstitial cystitis, ulcerative proctitis, and arthritis and herpes simplex infections $(5,8)$.

\section{Immunomodulating properties}

Studies demonstrated that LA, such as mepivacaine (MP), BP, and LD, induce innate immune system dysfunction, as indicated by reduced chemotactic ability, phagocytic ability, and superoxide anion production by neutrophils. Furthermore, LA also impair the activity of natural killer (NK) cells. There have been several studies about the effects of LA on immune function. BP and LD have modulatory effects and depress neutrofil chemotaxis and phagocytosis (27). Mitogen induced lymphocyte proliferation is also inhibited (28). Lymphocytes are capable of killing of a variety infectious or tumour derived target cells in vitro. Cell responsible for this activity are called NK cells. They are arrived from conventional T cells, B cells and macrophages. LA like LD and procaine affect the function of plasma membrane of human NK cell function (30). Impaired cytolysis by LA may be partially explained by the inhibition of binding of NK cells to target cells.

The concentrations of LA in human plasma reach $2.2 \mu \mathrm{g} / \mathrm{mL}$ (lidocaine), $2.53 \mu \mathrm{g} / \mathrm{mL}$ (mepivacaine), and $0.73 \mu \mathrm{g} / \mathrm{mL}$ (bupivacaine) after their epidural administration. In this study, the concentrations of LD, MP, and BP that suppressed monocyte HLA-DR expression were 1,1 , and $0.25 \mu \mathrm{g} / \mathrm{mL}$, respectively. These results suggest that LA suppress monocyte HLADR expression at clinically relevant concentrations. This suppressive effect might be stronger after local injection of these anaesthetics, because the local concentration might be larger than that in plasma. Careful attention must be paid to the effects of LA on the host-defense mechanisms in the clinical setting (21).

LD with epinephrine and prilocaine with felypressin were effective in significantly inhibiting adhesion, chemotaxis, phagocytosis, and the production of hydrogen peroxide by neutrophils and macrophages. Interestingly, LD with epinephrine potentiated the production of superoxide anion, whereas prilocaine with felypressine inhibited the production, irrespective of cells (3).

\section{Antimetastatic properties}

Experimental and clinical studies have shown that surgical trauma and stress affects the immune system including both the innate and adaptive immune responses. The break of immune homeostasis might enhance tumour growth and spread.

Investigations support idea that the using of intramuscular gene-gun therapy modified with BP can induce long-term antitumor immunity, and can provide the great advantage of inhibiting the disseminated tumour (1). Intramuscular administration of LA increases cell membrane permeability and enhance the expression of foreign genes in the muscles. Optimal interval after BP injection is 4 days after BP treatment into skeletal muscle. The other anaesthetic agents: LD and MP also enhance gene expression, but BP causes high membrane permeability. Innervation, blood supply and connective tissue are fully recovered after 7 days post BP treatment. 
Retrospective analysis suggests that paravertebral anaesthesia and analgesia for breast cancer surgery reduces the risk of recurrence or metastasis during the initial years of follow-up (9). There is ongoing research in Taiwan comparing local anaesthesia with general anaesthesia for breast cancer surgery and endometrial cancer surgery. Impaired cellular immunity after general anaesthesia has significant undesirable effects on tumour surveillance after breast surgery. The local block technique might avoid the surgery inducing neuroendocrine, metabolic, and cytokine responses, which will offer some advantages from better preservation of early postoperative cellular immune function and attenuate disturbance in the inflammatory mediators. Research focuses on the effects of regional anaesthesia on mediators that may be important in inflammatory response, tumour cell dissemination, deposition, and propagation in the early postoperative period.

\section{Systemic analgesic properties}

LA traditionally are administered for analgesia via epidural catheter, subcutaneous infiltration and regional nerve block procedures, but they have many potentially beneficial actions, especially when used as a continuous intravenous infusion. The use of intravenous LA infusions for postoperative pain control dates back to the 1940s when intravenous procaine was reported to provide analgesia for burns, abdominal surgery, and mastectomy. It was used in Stradins University Hospital during thoracic, abdominal and urological surgery. The use of parenteral LD for postoperative pain first was reported in 1961 by Bartlett (4).

Review briefly describes the anti-inflammatory properties of LA and discusses the benefits seen when used systemically for neuroprotection, postoperative ileus, decompression sickness, and glaucoma (31). There are only few studies dealing with systemic injection of LA. Therefore it is difficult to make a difference between the direct systemic effects of LA and the effects of epidural anaesthesia. There is a potential for an improved outcome after surgical procedures that is associated with significant perioperative stress reduction when LA are used. May be patients with contraindications to regional anaesthesia should receive LA intravenously for the surgical procedure. There is growing evidence that some patients would benefit from systemically administered LA (10).

LA infused intravenously to yield plasma concentrations far below those that block normal action potentials, yet that are frequently effective at reversing neuropathic pain. Thus, LA modify a variety of neuronal membrane channels and receptors, leading to what is probably a synergistic mixture of analgesic mechanisms to achieve effective clinical analgesia (32).

Group of Herroeder injected an intravenous LD bolus $1.5 \mathrm{mg} / \mathrm{kg}$ and followed by infusion $2 \mathrm{mg} / \mathrm{min}$. Gastrointestinal motility, pain scores and length of hospital stay were studied. Results were very promising: perioperative intravenous LD not only improved gastrointestinal motility but also shortened length of hospital stay significantly. Anti-inflammatory activity modulating the surgery-induced stress response may be one potential mechanism. Systemic LD may thus provide an additional approach to improve outcome for patients not suitable for centroaxial anaesthesia (11, 12, and 16). Acute rehabilitation after laparoscopic colectomy using intravenous LD gives similar outcomes to those reported using epidural analgesia (20).

LD infusions given to patients undergoing total hip arthroplasty in a manner similar to infusions given in visceral studies produced no opioid-sparing, significant difference in pain ratings, tactile pain thresholds, or maximal degree of active hip-flexion, which raises questions about the effectiveness of LD infusions for somatic pain (22).

Many clinicians may hesitate to use intravenous LD, fearing adverse cardiac and neurologic effects. LD is contraindicated in patients with cardiovascular instability, those concomitantly using a-agonists or $\beta$ blockers, and in patients with allergies to other amide LA (BP) (10). Perioperative intravenous administration of LD is an old technique that consistently has been shown to contribute to analgesia without respiratory depression, significant nausea, and other side effects common with opioid analgesics. A closer examination to determine an optimal regimen after visceral operations and its potential for use in other postoperative pain states is warranted.

But employing all positive properties of LA, we should take in account their general toxicity in large doses, and recently mentioned myotoxicity and effect on wound healing after local administration.

\section{Myotoxicity of local anaesthetics}

Clinically relevant skeletal muscle toxicity is probably a rare and rather unknown side effect of LA drugs. Nevertheless, skeletal muscle damage has to be considered a potentially serious complication of local and regional anaesthesia. Intramuscular injections of LA regularly result in striated muscle damage and myonecrosis, with a drug-specific and dose-dependent rate of toxicity (33). Subcellular pathomechanisms of LA myotoxicity are still not completely revealed. However, excessively increased intracellular Ca levels have been shown to have the key role in myocyte injury. Studying BP and RP both LA induced morphologically identical patterns of calcific myonecrosis, formation of scar tissue, and a marked rate of fiber regeneration. However, BP effects were constantly more pronounced than those of RP.

Active oxidative metabolism is a key determinant in $\mathrm{BP}$ toxicity, that BP myotoxicity is a relevant model of mitochondrial dysfunction involving the permeability transition pore and Ca dysregulation (18).

\section{Wound healing impairing}

LD and BP impair wound healing, but the mechanism of this side effect has not clearly understood. The phospholipid messenger lysophosphatide is released from activated platelets and induces fibroblast and smooth muscle proliferation. Lysophosphatide signalling was inhibited in the presence of LA and it may play a role in wound healing (23) 
From other papers LA can adversely affect cell growth in vitro. Their effects on wound healing are controversial. Low concentrations of LD, as would be seen in plasma after spinal, epidural, or plexus anesthesia, do not significantly affect multiplication of fibroblasts. Higher doses of LD arrest cell multiplication at the S-phase of the growth cycle by an extremely potent inhibitor of cell multiplication. Higher concentrations, as would be seen after tissue infiltration, severely inhibit fibroblast multiplication and thus may impair wound healing (7).

\section{CONCLUSIONS}

New LA should offer, in addition to a potential decrease in cardiac toxicity and improved separation of motor and sensory blockade, further benefits because of its inflammatory and immune modulating action, as compared with racemic BP and other appropriate local anaesthetics. Further research should address the question of how much inflammatory or immune modulation is necessary to prevent inflammatory response with lack of the sodium channel-blocking property.

We stand up for extensive use of LA in abdominal surgery, oncologic surgery and taking an advantage from their systemic qualities avoiding local toxic effects.

\section{Conflict of interest: None}

\section{REFERENCES}

1. Ajiki T, Murakami T, Kobayashi Y, Hakamata Y, Wang J, Inoue S, Ohtsuki M, Nakagawa H, Kariya Y, Hoshino Y, Kobayashi E. Long-lasting gene expression by particle-mediated intramuscular transfection modified with bupivacaine: combinatorial gene therapy with IL-12 and IL-18 cDNA against rat sarcoma at a distant site // Cancer Gene Ther, 2003; 10:318 - 329

2. Aydin ON, Evigor M, Aydin M. Antimicrobial activity of ropivacaine and other local anaesthetics // Eur J Anaesth, 2001; 18:687 - 694

3. Azuma Y, Ohura K. Immunological modulation by lidocaine-epinephrine and prilocaine-felypressin on the functions related to natural immunity in neutrophils and macrophages // Curr Drug Targ Imm Endocr Metabol Disord, 2004; 4:29 - 36

4. Bartlett EE, Hutaserani O. Xylocaine for the relief of postoperative pain // Anesth Analg, 1961; 40: $296-304$

5. Cassuto J, Sinclair R, Bonderovic M. Antiinflammatory properties of local anaesthetics and their present and potential clinical implications // Acta Anaesth Scand, 2006; 50:265 - 282

6. Daisy TJ, Gail KW. Drug interactions: lipoxygenase inhibitors interfere with ropivacaine induced vasoconstriction // Can J Anesth, 2009; 56: $279-283$

7. Desai D, Kojima K, Vacanti CA, Kodama S. Lidocaine inhibits NIH-3T3 cell multiplication by increasing the expression of cyclin-dependent kinase inhibitor 1A (p21) // Anesth Analg, 2008; 107:1592 - 1597

8. Doran C, Ji X. The anti-inflammatory effect of local anaesthetics // The Pain Llinic, 2007; 19(5): $207-213$

9. Exadaktylos AK, Buggy DJ, Moriarty, Mascha E, SesslerDI. Cananesthetictechniqueforprimarybreast cancer surgery affect recurrence or metastasis? // Anesthesiology, 2006; 105(4):660 - 664

10. Gordon D, Schroeder M. Intravenous lidocaine for postoperative analgesia: renewed interest in an old strategy // APS Bulletin, 2008; 18(3)

11. Groudine SB, Fisher HAG, Kaufman RP. Intravenous lidocaine speeds the return of bowel function, decreases postoperative pain, and shortens hospital stay in patients undergoing radical retropubic prostatectomy // Anesth Analg, 1998; 86:235 - 239

12. Herroeder S, Pecher S, Schönherr ME, Kaulitz G, Hahnenkamp K, Friess J, Böttiger BD, Bauer H, Dijkgraaf MGW, Durieux ME, Hollmann MW. Systemic Lidocaine shortens length of hospital stay after colorectal surgery // Ann Surg, 2007; 246(2): $192-200$

13. Hollmann MW, Durieux ME. Local anesthetics and the inflammatory response // Anesthesiology, 2000; 93:858 - 75

14. Hollmann MW, Gross A, Jelacin N, Durieux ME. Local anesthetic effects on priming and activation of human neutrophils // Anesthesiology, 2001; 95: $113-22$

15. Hollmann MW, Kurz K, Herroeder S, Struemper D, Hahnenkamp K, Berkelmans N, den Bakker CG, Durieux ME. The Effects of S-, R-, and Racemic Bupivacaine on Lysophosphatidate-Induced Priming of Human Neutrophils // Anesth Analg, 2003; 97:1053- 1058

16. Hollmann MW, Strumper D, Durieux ME. The poor man's epidural: systemic local anesthetics for improving postoperative outcomes // Med Hypotheses, 2004; 63:386 - 389

17. Honnemann CV, Heyse TJ, Mollhoff T, Hahnenkamp K, Berning S, Hinder F, Linck B, Schmitz W, van Aken, H. The inhibitory effect of bupivacaine on prostaglandin E (2) (EP (1)) receptor functionig: mechanism of action // Anesth Analg, 2001; 93: $628-634$

18. Irwin W, Fontaine E, Agnolucci L, Penzo D, Betto R, Bortolotto S, Reggiani C, Salviati J, Bernardi P. Bupivacaine myotoxicity is mediated by mitochondria // J Biol Chem, 2002; 2779(14): $12221-12227$

19. Johnson SM, Sain John BE, Dine AP. Local anaesthetics as antimicrobials agents: a review // Surg Infect (Larchmt), 2008; 9(2):205 - 213

20. Kaba A, Detroz BJ, Laurent SR. Acute rehabilitation program after laparoscopic colectomy using intravenous lidocaine // Acta Chir Belg, 2005; 105:53- 58

21. Kawasaki T, Kawasaki C, Ogata M, Shimegatsu O. The effect of local anesthetics on monocyte mCD14 and human leukocyte antigen-DR expression // Anesth Analg, 2004; 98: 1024 - 1029

22. Martin F, Cherif K, Gentilli ME. Lack of impact of intravenous lidocaine on analgesia, functional 
recovery, and nociceptive pain threshold after total hip arthroplasty // Anesth, 2008; 109:118 - 123

23. Nietgen GW, Chan CK, Durieux ME. Inhibition of Lysophosphatidate Signaling by Lidocaine and Bupivacaine // Anesthesiology, 1997; 86 (5): 1112-1119

24. Pietruck C, Grond S, Xie GX, Palmer P. Local anaethetics differentialy inhibit sympathetic Neuron mediated and $\mathrm{C}$ fiber mediated synovial plasma extravasation // Anesth Analg, 2003; 96: $1397-1402$

25. Ploppa A, Kiefer RT, Krueger WA, Unertl KE, Durieux ME. Local anesthetics time-dependently inhibit Staphylococcus aureus phagocytosis, oxidative burst and CDllb expression by human Neutrophils // Reg Anesth Pain Med, 2008; 33(4):297-303

26. Rosenberg PH, Renkonen OV. Antimicrobial activity of bupivacaine and morphine // Anesthesiology, $1985 ; 62: 178-179$

27. Ramus GV, Cezano L, Barbalonga A. Different concentrations of local anaesthetics have different modes of action on human lymphocytes // Agents Actions, 1983; 13:333 - 341

28. Sinclair R, Ericcson AS, Greizer G. Inhibitory effects of amide local anaesthetics on stimulus-induced human leucocyte metabolic activation, LTB4 release and Il-1 secretion in vitro // Acta Anaest Scand, $1993 ; 37: 159-165$
29. Sung HJ, Sohn JT, Hwang EM. Direct effect of ropivacaine involves lipoxygenase pathway activation in rat aortic smooth muscle // Can J Anest, 2009; 56:4

30. Takagi S, Kitagawa S, Oshimi K, Takaku F, Miura Y. Effect of local anaesthetics on human natural cell activity // Clin Exp Immunol, 1983; 53:477 - 481

31. Wright JL, Durieux ME, Groves DS. A brief review of innovative uses for local anesthetics // Cur Op Anaesth, 2008; 21(5):651 - 656

32. Yanagidate F, Strichart GR. Analgesia // In: Handbook of experimental Pharmacology. Volume 177, Springer Berlin Heidelberg, 2007; 95-127

33. Zink W, Bohl JRE, Hacke N, Sinner B, Martin E, Graf B. The long term myotoxic effects of bupivacaine and ropivacaine after continuous peripheral nerve blocks // Anesth Analg, 2005; 101:548 - 554

\section{Address:}

Iveta Golubovska

Hospital of Traumatology and Orthopaedics,

Duntes str. 12, Riga, LV1005, Latvia

Email: ivetagolubovska@gmail.com 